\title{
On the Generalized Fibonacci-circulant-Hurwitz numbers
}

\author{
Ömür Deveci ${ }^{1}$, Zafer Adıgüzel ${ }^{2}$ and Taha Doğan ${ }^{3}$ \\ ${ }^{1}$ Department of Mathematics, Faculty of Science and Letters \\ Kafkas University, 36100, Turkey \\ e-mail: odeveci36@hotmail.com \\ ${ }^{2}$ Department of Mathematics, Faculty of Science and Letters \\ Kafkas University, 36100, Turkey \\ e-mail: zafer-adiguzel36@hotmail.com \\ ${ }^{3}$ Department of Mathematics, Faculty of Science and Letters \\ Kafkas University, 36100, Turkey \\ e-mail: tahadogan8636@gmail.com
}

Received: 28 May 2019 Revised: 22 December $2019 \quad$ Accepted: 30 December 2019

\begin{abstract}
The theory of Fibonacci-circulant numbers was introduced by Deveci et al. (see [5]). In this paper, we define the Fibonacci-circulant-Hurwitz sequence of the second kind by Hurwitz matrix of the generating function of the Fibonacci-circulant sequence of the second kind and give a fair generalization of the sequence defined, which we call the generalized Fibonacci-circulantHurwitz sequence. First, we derive relationships between the generalized Fibonacci-circulantHurwitz numbers and the generating matrices for these numbers. Also, we give miscellaneous properties of the generalized Fibonacci-circulant-Hurwitz numbers such as the Binet formula, the combinatorial, permanental, determinantal representations, the generating function, the exponential representation and the sums.
\end{abstract}

Keywords: Fibonacci-circulant-Hurwitz Sequence, Circulant matrix, Hurwitz matrix, Representation.

2010 Mathematics Subject Classification: 11K31, 11B50, 11C20, 20 D60. 


\section{Introduction}

The $k$-step Fibonacci sequence $\left\{F_{n}^{k}\right\}$ is defined by initial values $F_{0}^{k}=F_{1}^{k}=F_{k-2}^{k}=0, F_{k-1}^{k}=1$ and recurrence relation

$$
F_{n+k}^{k}=F_{n+k-1}^{k}+F_{n+k-2}^{k}+\cdots+F_{n}^{k} \text { for } n \geq 0 .
$$

For detailed information about the $k$-step Fibonacci sequence, see $[9,21]$.

In [5], Deveci et al. defined the Fibonacci-circulant sequence of the second kind as shown:

$$
x_{1}^{2}=\cdots=x_{4}^{2}=0, x_{5}^{2}=1 \text { and } x_{n}^{2}=-x_{n-3}^{2}+x_{n-4}^{2}-x_{n-5}^{2} \text { for } n \geq 6 .
$$

Note that the characteristic polynomial of the Fibonacci-circulant sequence of the second kind is as follows:

$$
f(x)=-x^{5}+x^{2}+x-1 .
$$

Let an $n$-th degree real polynomial $f$ be given by

$$
f^{2}(x)=c_{0} x^{n}+c_{1} x^{n-1}+\cdots+c_{n-1} x+c_{n} .
$$

In [8], the Hurwitz matrix $H_{n}=\left[h_{i, j}\right]_{n \times n}$ associated to the polynomial $f$ was defined as shown:

$$
H_{n}=\left[\begin{array}{ccccccccc}
c_{1} & c_{3} & c_{5} & \cdots & \cdots & \cdots & 0 & 0 & 0 \\
c_{0} & c_{2} & c_{4} & \cdots & \cdots & \cdots & \vdots & \vdots & \vdots \\
0 & c_{1} & c_{3} & \cdots & \cdots & \cdots & \vdots & \vdots & \vdots \\
\vdots & c_{0} & c_{2} & \ddots & \ddots & \ddots & 0 & \vdots & \vdots \\
\vdots & 0 & c_{1} & \ddots & \ddots & \ddots & c_{n} & \vdots & \vdots \\
\vdots & \vdots & c_{0} & \ddots & \ddots & \ddots & c_{n-1} & 0 & \vdots \\
\vdots & \vdots & 0 & \cdots & \cdots & \cdots & c_{n-2} & c_{n} & \vdots \\
\vdots & \vdots & \vdots & \cdots & \cdots & \cdots & c_{n-3} & c_{n-1} & 0 \\
0 & 0 & 0 & \cdots & \cdots & \cdots & c_{n-4} & c_{n-2} & c_{n}
\end{array}\right]
$$

Consider the $k$-step homogeneous linear recurrence sequence $\left\{a_{n}\right\}$,

$$
a_{n+k}=c_{0} a_{n}+c_{1} a_{n+1}+\cdots+c_{k-1} a_{n+k-1},
$$

where $c_{0}, c_{1}, \ldots, c_{k-1}$ are real constants. In [9], Kalman derived a number of closed-form formulas for the sequence $\left\{a_{n}\right\}$ by matrix method as follows:

$$
A^{n}\left[\begin{array}{c}
a_{0} \\
a_{1} \\
\vdots \\
a_{k-1}
\end{array}\right]=\left[\begin{array}{c}
a_{n} \\
a_{n+1} \\
\vdots \\
a_{n+k-1}
\end{array}\right]
$$


, where

$$
A=\left[a_{i, j}\right]_{k \times k}=\left[\begin{array}{cccccc}
0 & 1 & 0 & \cdots & 0 & 0 \\
0 & 0 & 1 & \cdots & 0 & 0 \\
0 & 0 & 0 & \cdots & 0 & 0 \\
\vdots & \vdots & \vdots & \ddots & \vdots & \vdots \\
0 & 0 & 0 & \cdots & 0 & 1 \\
c_{0} & c_{1} & c_{2} & & c_{k-2} & c_{k-1}
\end{array}\right] .
$$

Number theoretic properties such as these obtained from Fibonacci numbers relevant to this paper have been studied by many authors $[1,4,7,11,12,20,23,27,28]$. Now we define the generalized Fibonacci-circulant-Hurwitz numbers and then, we obtain their miscellaneous properties using the generating matrix and the generating function of these numbers.

\section{Significance}

As it is well-known that recurrence sequences, circulant matrix and Hurwitz matrix appear in modern research in many fields from mathematics, physics, computer science, architecture to nature and art (see, for example, $[6,10,13,14,17,18,19,22,24,25,26])$. This paper is expanded the concept to the generalized Fibonacci-circulant-Hurwitz sequence which is defined by using circulant and Hurwitz matrices.

\section{The main resutls}

By the polynomial $f^{2}(x)$, we can write the following Hurwitz matrix:

$$
M^{2}=\left[\begin{array}{ccccc}
0 & 1 & -1 & 0 & 0 \\
1 & 0 & 1 & 0 & 0 \\
0 & 0 & 1 & -1 & 0 \\
0 & 1 & 0 & 1 & 0 \\
0 & 0 & 1 & 0 & -1
\end{array}\right] \text {. }
$$

Using the matrix $M^{2}$, we define the Fibonacci-circulant-Hurwitz sequence of the second kind as shown:

$$
a_{1}^{2}=\cdots=a_{4}^{2}=0, a_{5}^{2}=1 \text { and } a_{n+1}^{2}=-a_{n}^{2}+a_{n-1}^{2}+a_{n-2}^{2}+a_{n-4}^{2} \text { for } n \geq 5 .
$$

Now we consider a new sequence which is a generalized form of the the Fibonacci-circulantHurwitz sequence of the second kind and is called the generalized Fibonacci-circulant-Hurwitz sequence. The sequence is defined by integer constants $a_{1}^{k}=\cdots=a_{k-1}^{k}=0, a_{k}^{k}=1$ and the recurrence relation

$$
a_{n+1}^{k}=-a_{n}^{k}+a_{n-1}^{k}+\cdots+a_{n-k+3}^{k}+a_{n-k+1}^{k}
$$

for $n \geq k$, where $k$ is a positive integer such that $k \geq 4$. 
From (1), we may write the following matrix:

$$
M_{k}=\left[m_{i, j}\right]_{k \times k}=\left[\begin{array}{ccccccc}
-1 & 1 & 1 & \cdots & 1 & 0 & 1 \\
1 & 0 & 0 & \cdots & 0 & 0 & 0 \\
0 & 1 & 0 & \cdots & 0 & 0 & 0 \\
0 & 0 & 1 & \cdots & 0 & 0 & 0 \\
\vdots & \vdots & \vdots & \ddots & \vdots & \vdots & \vdots \\
0 & 0 & 0 & \cdots & 1 & 0 & 0 \\
0 & 0 & 0 & \cdots & 0 & 1 & 0
\end{array}\right] .
$$

The matrix $M_{k}$ is called the generalized Fibonacci-circulant-Hurwitz matrix.

Note that $\operatorname{det}\left(M_{k}\right)=(-1)^{k+1}$ for $k \geq 4$.

By induction on $n$, we get

$$
\begin{gathered}
\left(M_{4}\right)^{n}=\left[\begin{array}{cccc}
a_{n+4}^{4} & a_{n+3}^{4}+a_{n+1}^{4} & a_{n+2}^{4} & a_{n+3}^{4} \\
a_{n+3}^{4} & a_{n+2}^{4}+a_{n}^{4} & a_{n+1}^{4} & a_{n+2}^{4} \\
a_{n+2}^{4} & a_{n+1}^{4}+a_{n-1}^{4} & a_{n}^{4} & a_{n+1}^{4} \\
a_{n+1}^{4} & a_{n}^{4}+a_{n-1}^{4} & a_{n-1}^{4} & a_{n}^{4}
\end{array}\right], \\
\left(M_{5}\right)^{n}=\left[\begin{array}{ccccc}
a_{n+5}^{5} & a_{n+6}^{5}+a_{n+5}^{5} & a_{n+4}^{5}+a_{n+2}^{5} & a_{n+3}^{5} & a_{n+4}^{5} \\
a_{n+4}^{5} & a_{n+5}^{5}+a_{n+4}^{5} & a_{n+3}^{5}+a_{n+1}^{5} & a_{n+2}^{5} & a_{n+3}^{5} \\
a_{n+3}^{5} & a_{n+4}^{5}+a_{n+3}^{5} & a_{n+2}^{5}+a_{n}^{5} & a_{n+1}^{5} & a_{n+2}^{5} \\
a_{n+2}^{5} & a_{n+3}^{5}+a_{n+2}^{5} & a_{n+1}^{5}+a_{n-1}^{5} & a_{n}^{5} & a_{n+1}^{5} \\
a_{n+1}^{5} & a_{n+2}^{5}+a_{n+1}^{5} & a_{n}^{5}+a_{n-2}^{5} & a_{n-1}^{5} & a_{n}^{5}
\end{array}\right]
\end{gathered}
$$

and

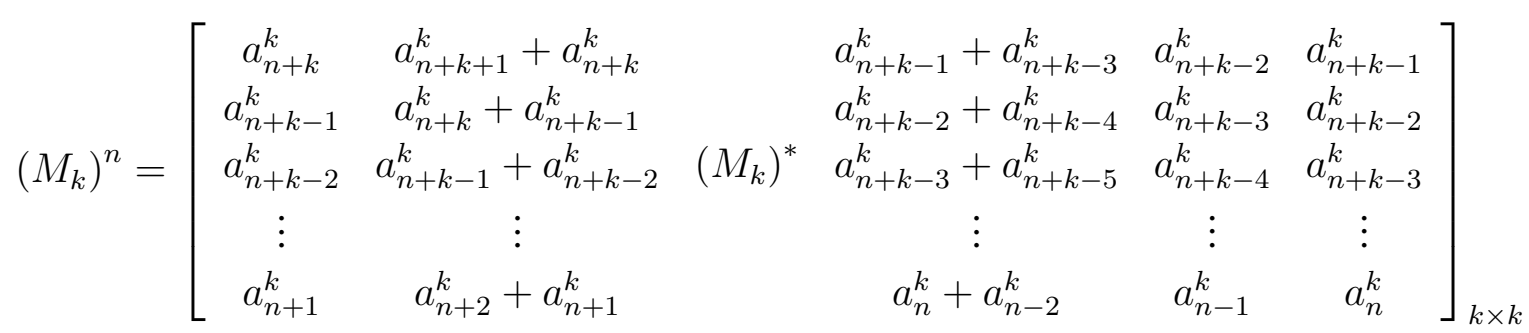

for $k \geq 6$, where $\left(M_{k}\right)^{*}$ is a matrix with $k$ row and $k-5$ column given below:

$$
\left[\begin{array}{cccc}
a_{n+k-1}^{k}+\cdots+a_{n+4}^{k}+a_{n+2}^{k} & a_{n+k-1}^{k}+\cdots+a_{n+5}^{k}+a_{n+3}^{k} & \cdots & a_{n+k-1}^{k}+a_{n+k-2}^{k}+a_{n+k-4}^{k} \\
a_{n+k-2}^{k}+\cdots+a_{n+3}^{k}+a_{n+1}^{k} & a_{n+k-2}^{k}+\cdots+a_{n+4}^{k}+a_{n+2}^{k} & \cdots & a_{n+k-2}^{k}+a_{n+k-3}^{k}+a_{n+k-5}^{k} \\
\vdots & \vdots & \ddots & \vdots \\
a_{n}^{k}+\cdots+a_{n-k+4}^{k}+a_{n-k+2}^{k} & a_{n}^{k}+\cdots+a_{n-k+5}^{k}+a_{n-k+3}^{k} & \cdots & a_{n}^{k}+a_{n-1}^{k}+a_{n-3}^{k}
\end{array}\right] .
$$

Lemma 3.1. The characteristic equation of all the generalized Fibonacci-circulant-Hurwitz numbers $x^{k}+x^{k-1}-x^{k-2}-\cdots-x^{2}-1=0$ does not have multiple roots for $k \geq 4$. 
Proof. Let $f(x)=x^{k}+x^{k-1}-x^{k-2}-\cdots-x^{2}-1$. We easily see that $f(1) \neq 1$. Consider $h(x)=(x-1) f(x)$. Since $f(1) \neq 1,1$ is root but not a multiple root of $h(x)$. Assume that $u$ a multiple root of $h(x)$. Then $h(u)=0$ and $h^{\prime}(u)=0$. So we get

$$
(1-k) u^{4}+k u^{3}+(k-7) u^{2}+(4-2 k) u+2(k-1)=0 .
$$

Using appropriate softwares such as Wolfram Mathematica 10.0 [29], one can see that this last equation does not have a solution which is a contradiction. This contradiction proves that the equation $f(x)$ does not have multiple roots.

If $x_{1}, x_{2}, \ldots, x_{k}$ are the eigenvalues of the generalized Fibonacci-circulant-Hurwitz matrix $M_{k}$, then by Lemma 3.1, it is known that $x_{1}, x_{2}, \ldots, x_{k}$ are distinct. Let a $k \times k$ Vandermonde matrix $V^{k}$ be given by

$$
V^{k}=\left[\begin{array}{cccc}
\left(x_{1}\right)^{k-1} & \left(x_{2}\right)^{k-1} & \cdots & \left(x_{k}\right)^{k-1} \\
\left(x_{1}\right)^{k-2} & \left(x_{2}\right)^{k-2} & \cdots & \left(x_{k}\right)^{k-2} \\
\vdots & \vdots & \ddots & \vdots \\
x_{1} & x_{2} & \cdots & x_{k} \\
1 & 1 & \cdots & 1
\end{array}\right] .
$$

Now assume that $W^{k}(i)$ is a $(p+2) \times 1$ matrix as shown:

$$
W^{k}(i)=\left[\begin{array}{c}
\left(x_{1}\right)^{n+k-i} \\
\left(x_{2}\right)^{n+k-i} \\
\vdots \\
\left(x_{p+2}\right)^{n+k-i}
\end{array}\right]
$$

and $V^{k}(i, j)$ is a $k \times k$ matrix derived from the Vandermonde matrix $V^{k}$ by replacing the $j$-th column of $V^{k}$ by matrix $W^{k}(i)$.

Now we give the Binet formulas for the generalized Fibonacci-circulant-Hurwitz numbers by the following Theorem.

Theorem 3.1. Let $k$ be a positive integer such that $k \geq 4$ and let $\left(M_{k}\right)^{\alpha}=\left[m_{i, j}^{(\alpha)}\right]$ for $\alpha \geq 1$, then

$$
m_{i, j}^{(\alpha)}=\frac{\operatorname{det} V^{k}(i, j)}{V^{k}} .
$$

Proof. Since the eigenvalues of the generalized Fibonacci-circulant-Hurwitz matrix $M_{k}$ are distinct, $M_{k}$ is diagonalizable. Then, we may write $M_{k} V^{k}=V^{k} D_{k}$, where $D_{k}=\operatorname{diag}\left(x_{1}, x_{2}, \ldots\right.$, $\left.x_{k}\right)$. Since $\operatorname{det} V^{k} \neq 0$, we get

$$
\left(V^{k}\right)^{-1} M_{k} V^{k}=D_{k} .
$$

It will thus be seen that the matrices $M_{k}$ and $D_{k}$ are similar. Then we can write the matrix equation $\left(M_{k}\right)^{\alpha} V^{k}=V^{k}\left(D_{k}\right)^{\alpha}$ for $\alpha \geq 1$. Since $\left(M_{k}\right)^{\alpha}=\left[m_{i, j}^{(\alpha)}\right]$, we get

$$
\left\{\begin{array}{c}
m_{i, 1}^{(\alpha)}\left(x_{1}\right)^{k-1}+m_{i, 2}^{(\alpha)}\left(x_{1}\right)^{k-2}+\cdots+m_{i, k}^{(\alpha)}=\left(x_{1}\right)^{\alpha+k-i} \\
m_{i, 1}^{(\alpha)}\left(x_{2}\right)^{k-1}+m_{i, 2}^{(\alpha)}\left(x_{2}\right)^{k-2}+\cdots+m_{i, k}^{(\alpha)}=\left(x_{2}\right)^{\alpha+k-i} \\
\vdots \\
m_{i, 1}^{(\alpha)}\left(x_{k}\right)^{k-1}+m_{i, 2}^{(\alpha)}\left(x_{k}\right)^{k-2}+\cdots+m_{i, k}^{(\alpha)}=\left(x_{k}\right)^{\alpha+k-i}
\end{array}\right.
$$


So we conclude that

$$
m_{i, j}^{(\alpha)}=\frac{\operatorname{det} V^{k}(i, j)}{V^{k}}
$$

for each $i, j=1,2, \ldots, k$.

Thus by Theorem 3.1 and the matrix $\left(M_{k}\right)^{n}$, we have the following useful results.

Corollary 3.1. Let $a_{n}^{k}$ be the $n$-th element of the generalized Fibonacci-circulant-Hurwitz sequence, then

$$
a_{n}^{k}=\frac{\operatorname{det} V^{k}(k, k)}{V^{k}}=\frac{\operatorname{det} V^{k}(k-1, k-1)}{V^{k}}
$$

for $k \geq 4$.

Now we consider the combinatorial representations for all the generalized Fibonacci-circulantHurwitz numbers.

Let a $k \times k$ companion matrix $C\left(c_{1}, c_{2}, \ldots, c_{k}\right)$ be given by

$$
C\left(c_{1}, c_{2}, \ldots, c_{k}\right)=\left[\begin{array}{cccc}
c_{1} & c_{2} & \cdots & c_{k} \\
1 & 0 & \cdots & 0 \\
\vdots & \ddots & \ddots & \vdots \\
0 & \cdots & 1 & 0
\end{array}\right]
$$

For more details on the companion type matrices, see $[15,16]$.

Theorem 3.2 (Chen and Louck [3]). The $(i, j)$ entry $c_{i, j}^{(\alpha)}\left(c_{1}, c_{2}, \ldots, c_{k}\right)$ in the matrix $C^{\alpha}\left(c_{1}, c_{2}, \ldots, c_{k}\right)$ is given by the following formula:

$$
c_{i, j}^{(\alpha)}\left(c_{1}, c_{2}, \ldots, c_{k}\right)=\sum_{\left(t_{1}, t_{2}, \ldots, t_{k}\right)} \frac{t_{j}+t_{j+1}+\cdots+t_{k}}{t_{1}+t_{2}+\cdots+t_{k}} \times\left(\begin{array}{c}
t_{1}+\cdots+t_{k} \\
t_{1}, \ldots, t_{k}
\end{array}\right) c_{1}^{t_{1}} \cdots c_{k}^{t_{k}}
$$

where the summation is over nonnegative integers satisfying $t_{1}+2 t_{2}+\cdots+k t_{k}=\alpha-i+j$, $\left(\begin{array}{c}t_{1}+\cdots+t_{k} \\ t_{1}, \ldots, t_{k}\end{array}\right)=\frac{\left(t_{1}+\cdots+t_{k}\right) !}{t_{1} ! \cdots t_{k} !}$ is a multinomial coefficient, and the coefficients in (4) are defined to be 1 if $\alpha=i-j$.

Corollary 3.2. Let $k$ be a positive integer such that $k \geq 4$ and let $a_{n}^{k}$ be the $n$-th element of the generalized Fibonacci-circulant-Hurwitz sequence, then

$$
\begin{aligned}
a_{n}^{k} & =\sum_{\left(t_{1}, t_{2} \ldots, t_{k}\right)} \frac{t_{k}}{t_{1}+t_{2}+\cdots+t_{k}} \times\left(\begin{array}{c}
t_{1}+\cdots+t_{k} \\
t_{1}, \ldots, t_{k}
\end{array}\right) \\
& =\sum_{\left(t_{1}, t_{2} \ldots, t_{p+2}\right)} \frac{t_{k-1}+t_{k}}{t_{1}+t_{2}+\cdots+t_{k}} \times\left(\begin{array}{c}
t_{1}+\cdots+t_{k} \\
t_{1}, \ldots, t_{k}
\end{array}\right)
\end{aligned}
$$

where the summation is over nonnegative integers satisfying $t_{1}+2 t_{2}+\cdots+k t_{k}=n$.

Proof. In Theorem 3.2, if we choose $i=j=k$ and $i=j=k-1$, then the proof is immediately seen from (3). 
Definition 3.1. An $u \times v$ real matrix $A=\left[a_{i, j}\right]$ is called a contractible matrix in the $n$-th column (resp. row) if the $n$-th column (resp. row) contains exactly two non-zero entries.

Let $x_{1}, x_{2}, \ldots, x_{u}$ be row vectors of the matrix $A$. If $A$ is contractible in the $n$-th column such that $a_{\tau, n} \neq 0, a_{\sigma, n} \neq 0$ and $\tau \neq \sigma$, then the $(u-1) \times(v-1)$ matrix $A_{\tau, \sigma: n}$ obtained from $A$ by replacing the $\tau$-th row with $a_{\tau, n} x_{\sigma}+a_{\sigma, n} x_{\tau}$ and deleting the $\sigma$-th row. We call the $n$-th column the contraction in the $n$-th column relative to the $\tau$-th row and the $\sigma$-th row.

In [2], it was shown that $\operatorname{per}(A)=\operatorname{per}(B)$ if $A$ is a real matrix of order $u>1$ and the matrix $B$ is a contraction of $A$.

Let $u \geq k$ and let a $u \times u$ super-diagonal matrix $N_{u}^{k}=\left[n_{i, j}^{k}\right]$ be given by

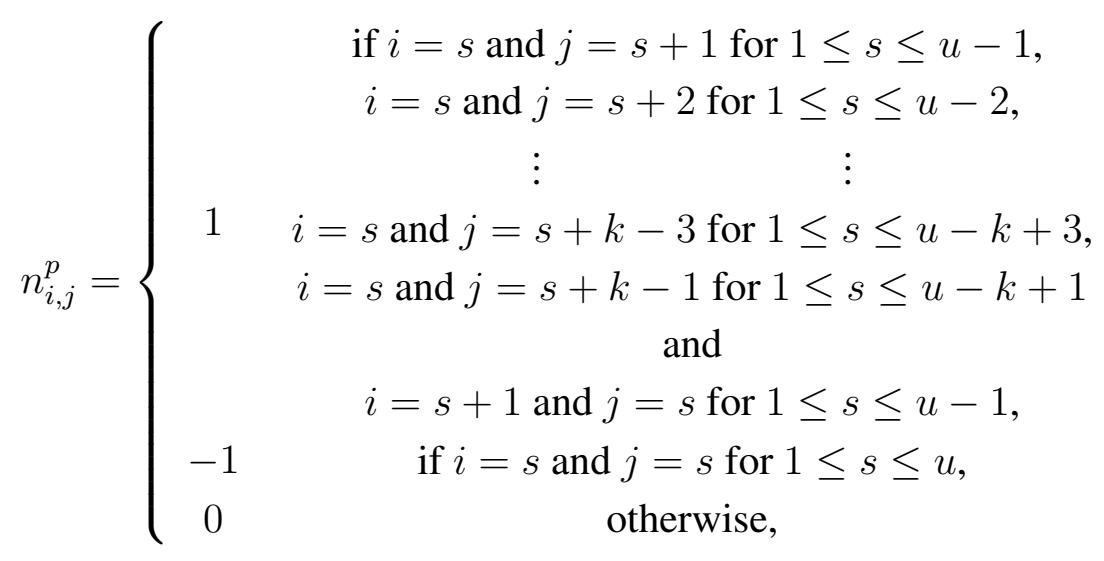

where $k \geq 4$.

Now we give the permanental representations for the generalized Fibonacci-circulant-Hurwitz numbers by the following Theorems.

Theorem 3.3. Let $a_{n}$ be the $n$-th element of the generalized Fibonacci-circulant-Hurwitz sequence, then

$$
\operatorname{per}\left(N_{u}^{k}\right)=a_{u+k}^{k}
$$

for $u \geq k$.

Proof. The assertion may be proved by induction on $u$. Assume that the result hold for any integer grater than or equal to $k$. Then we show the equation holds for $u+1$. Expanding the $\operatorname{per}\left(N_{u}^{k}\right)$ by the Laplace expansion of permanent according to the first row gives us

$$
\operatorname{per}\left(N_{u+1}^{k}\right)=-\operatorname{per}\left(N_{u}^{k}\right)+\operatorname{per}\left(N_{u-1}^{k}\right)+\cdots+\operatorname{per}\left(N_{u-k+3}^{k}\right)+\operatorname{per}\left(N_{u-k+1}^{k}\right) .
$$

Since

$$
\operatorname{per}\left(N_{u}^{k}\right)=a_{u+k}^{k}, \operatorname{per}\left(N_{u-1}^{k}\right)=a_{u+k-1}^{k}, \ldots, \operatorname{per}\left(N_{u-k+3}^{k}\right)=a_{u+3}^{k}, \operatorname{per}\left(N_{u-k+1}^{k}\right)=a_{u+1}^{k},
$$

by using the recurrence relation of the generalized Fibonacci circulant-Hurwitz numbers, we obtain $\operatorname{per}\left(N_{u+1}^{k}\right)=a_{u+k+1}^{k}$. 
Suppose that $u>k$ and the $u \times u$ matrices $H_{u}^{k}=\left[h_{i, j}^{k}\right]$ and $T_{u}^{k}=\left[t_{i, j}^{k}\right]$ are defined by

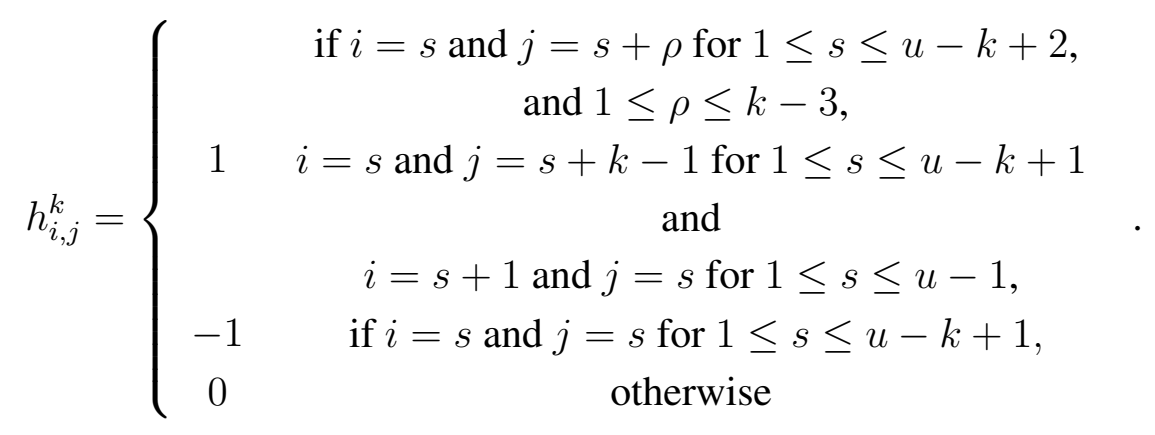

and

$$
T_{u}^{k}=\left[\begin{array}{cccccc}
1 & \cdots & 1 & 0 & \cdots & 0 \\
1 & & & & & \\
0 & & & H_{u-1}^{k} & & \\
\vdots & & & & & \\
0 & & & & &
\end{array}\right]
$$

$k \geq 4$.

Using the matrices $H_{u}^{k}=\left[h_{i, j}^{k}\right]$ and $T_{u}^{k}=\left[t_{i, j}^{k}\right]$ and the above results we can give more general permanental representations.

Theorem 3.4. For $u>k$,

$$
\operatorname{per}\left(H_{u}^{k}\right)=a_{u}^{k},
$$

and

$$
\operatorname{per}\left(T_{u}^{k}\right)=\sum_{\tau=0}^{u-1} a_{\tau}^{k}
$$

Proof. Consider the first part of the theorem. We prove this by the induction method. Suppose that the equation holds for $u>k$, then we show that the equation holds for $u+1$. If we expand the per $\left(H_{u}^{k}\right)$ by the Laplace expansion of permanent according to the first row, then we get

$$
\begin{aligned}
\operatorname{per}\left(H_{u+1}^{k}\right) & =-\operatorname{per}\left(H_{u}^{k}\right)+\operatorname{per}\left(H_{u-1}^{k}\right)+\cdots+\operatorname{per}\left(H_{u-k+3}^{k}\right)+\operatorname{per}\left(H_{u-k+1}^{k}\right) \\
& =-a_{u}^{k}+a_{u-1}^{k}+\cdots+a_{u-k+3}^{k}+a_{u-k+1}^{k} \\
& =a_{u+1}^{k} .
\end{aligned}
$$

Prove the second part of the theorem: Expanding the per $\left(T_{u}^{k}\right)$ with respect to the first row, we can write

$$
\operatorname{per}\left(T_{u}^{k}\right)=\operatorname{per}\left(T_{u-1}^{k}\right)+\operatorname{per}\left(H_{u-1}^{p}\right) .
$$

Thus, by the results and an inductive argument, the proof is easily seen. 
Using the definition of the generalized Fibonacci-circulant-Hurwitz numbers we find the generating function $g(x)$ as shown

$$
g(x)=\frac{x^{k}}{1+x-x^{2}-\cdots-x^{k-2}-x^{k}}
$$

where $k \geq 4$.

Now we investigate an exponential representation for the generalized Fibonacci-circulantHurwitz numbers.

Theorem 3.5. For $k \geq 4$, the generalized Fibonacci-circulant-Hurwitz numbers have the following exponential representation:

$$
g(x)=x^{k} \exp \left(\sum_{n=1}^{\infty} \frac{x^{n}}{n}\left(-1+x+\cdots+x^{k-3}+x^{k-1}\right)^{n}\right) .
$$

Proof. We consider the generating function $g(x)=\frac{x^{k}}{1+x-x^{2}-\cdots-x^{k-2}-x^{k}}$. Since

$$
\begin{gathered}
\ln g(x)=\ln \left(\frac{x^{k}}{1+x-x^{2}-\cdots-x^{k-2}-x^{k}}\right), \\
\ln g(x)=\ln x^{k}-\ln \left(1+x-x^{2}-\cdots-x^{k-2}-x^{k}\right)
\end{gathered}
$$

and

$$
\begin{aligned}
\ln \left(1+x-x^{2}-\cdots-x^{k-2}-x^{k}\right)= & -\left[x\left(-1+x+x^{2}+\cdots+x^{k-3}+x^{k-1}\right)\right. \\
& +\frac{1}{2} x^{2\left(-1+x+x^{2}+\cdots+x^{k-3}+x^{k-1}\right) 2}+\cdots \\
& \left.+\frac{1}{i} x^{i}\left(-1+x+x^{2}+\cdots+x^{k-3}+x^{k-1}\right)^{i}+\cdots\right],
\end{aligned}
$$

it is clear that

$$
\ln \frac{g(x)}{x^{k}}=\sum_{n=1}^{\infty} \frac{x^{n}}{n}\left(-1+x+\cdots+x^{k-3}+x^{k-1}\right)^{n} .
$$

Now we consider the sums of all the generalized Fibonacci-circulant-Hurwitz numbers. Let the $k \times k$ matrix $M_{k}$ be as in (2) and let the sums of the generalized Fibonacci-circulant-Hurwitz numbers from 1 to $n,(n>1)$ be denoted by $S_{n}$, that is,

$$
S_{n}=\sum_{i=1}^{n} a_{i}^{k}
$$

If we define the $(k+1) \times(k+1)$ matrix $Z_{k}$ as in the following form:

$$
Z_{k}=\left[\begin{array}{cccc}
1 & 0 & \cdots & 0 \\
1 & & & \\
0 & & M_{k} & \\
\vdots & & & \\
0 & & &
\end{array}\right]
$$


then by using induction on $n$, we may write

$$
\left(Z_{k}\right)^{n}=\left[\begin{array}{cccc}
1 & 0 & \cdots & 0 \\
S_{n+k-1} & & & \\
S_{n+k-1} & & \left(M_{k}\right)^{n} & \\
\vdots & & & \\
S_{n} & & &
\end{array}\right] .
$$

\section{Acknowledgements}

This Project was supported by the Commission for the Scientific Research Projects of Kafkas University, Project number 2017-FM-65.

\section{References}

[1] Atanassov, K. T., Atanassova, V. K., Shannon, A. G., \& Turner, J. (2002). New Visual Perspectives on Fibonacci Numbers, World Scientific.

[2] Brualdi, R. A., \& Gibson, P. M. (1997). Convex polyhedra of doubly stochastic matrices I: applications of permanent function, J. Combin. Theory, 22, 194-230.

[3] Chen, W. Y. C., \& Louck, J. C. (1996). The combinatorial power of the companion matrix, Linear Algebra Appl., 232, 261-278.

[4] Deveci, O. (2018). On the Fibonacci-circulant p-sequences, Util Math., 108, 107-124.

[5] Deveci, O., Karaduman, E., \& Campbell, C. M. (2017). The Fibonacci-circulant sequences and their applications, Iran J. Sci. Technol. Trans. Sci., 41 (4), 1033-1038.

[6] El Naschie, M. S. (2005). Deriving the essential features of standard model from the general theory of relativity, Chaos Solitons Fractals, 24 (4), 941-946.

[7] Gogin, N. D., \& Myllari, A. A. (2007). The Fibonacci-Padovan sequence and MacWilliams transform matrices, Program. Comput Softw published in Programmirovanie, 33 (2), 74-79.

[8] Hurwitz, A. (1895). Ueber die Bedingungen unter welchen eine gleichung nur Wurzeln mit negative reellen teilen besitzt, Mathematische Annalen, 46, 273-284.

[9] Kalman, D. (1982). Generalized Fibonacci numbers by matrix methods, Fibonacci Quart., 20 (1), 73-76.

[10] Kaluge, G. R. (2011). Penggunaan Fibonacci dan Josephus problem dalam algoritma enkripsi transposisi+substitusi, Makalah IF 3058 Kriptografi-Sem. II Tahun. 
[11] Kilic, E. (2008). The Binet formula, sums and representations of generalized Fibonacci p-numbers, European J. Combin, 29, 701-711.

[12] Kilic, E., \& Tasci, D. (2007). On the permanents of some tridiagonal matrices with applications to the Fibonacci and Lucas numbers, Rocky Mountain J. Math., 37 (6), 1953-1969.

[13] Kirchoof, B. K., \& Rutishauser, R. (1990). The phyllotaxy of costus (costaceae), Bot Gazette, 151 (1), 88-105.

[14] Kraus, F. J., Mansour, M., \& Sebek, M. (1996). Hurwitz Matrix for Polynomial Matrices, In Jeltsch R Mansour M (eds) Stability Theory ISNM International Series of Numerical Mathematics 121 Birkhäuser Basel.

[15] Lancaster, P., \& Tismenesky, M. (1985). The Theory of Matrices, Academic Press.

[16] Lidl, R., \& Niederreiter, H. (1986). Introduction to Finite Fields and Their Applications, Cambridge UP.

[17] Lipshitz, L., \& van der, A. (1990). Poorten AJ Rational functions, diagonals, automata and arithmetic, Number Theory (Banff, AB, 1988) de Grutyer, Berlin, 339-358.

[18] Mandelbaum, D. M. (1972). Synchronization of codes by means of Kautz's Fibonacci encoding, IEEE Transactions on Information Theory, 18 (2), 281-285.

[19] Matiyasevich, Y. V. (1993). Hilbert's Tenth Problem, MIT Press, Cambridge, MA.

[20] Shannon, A. G., \& Leyendekkers, J.V. (2011). Pythagorean Fibonacci patterns, Int. J. Math. Educ. Sci. Technol., 43 (4), 554-559.

[21] Sloane, N. J. A. Sequences A000045/M0692, A000073/M1074, A000078/M1108, A001591, A001622, A046698, A058265, A086088, and A118745 in The On-Line Encyclopedia of Integer Sequences.

[22] Spinadel, V. W. (1999). The family of metallic means, Vis Math, 1(3) Mathematical Institute SASA.

[23] Stakhov, A. P., \& Rozin, B. (2006). Theory of Binet formulas for Fibonacci and Lucas p-numbers, Chaos Solitons Fractals, 27 (5), 1162-1167.

[24] Stein, W. (1993). Modelling the evolution of Stelar architecture in Vascular plants, Int. J. Plant. Sci., 154 (2), 229-263.

[25] Stewart, I. (1996). Tales of neglected number, Sci. Amer., 274, 102-103.

[26] Stroeker, R. J. (1988). Brocard Points, Circulant Matrices, and Descartes Folium, Math. Mag., 61 (3), 172-187. 
[27] Tasci, D., \& Firengiz, M. C. (2010). Incomplete Fibonacci and Lucas p-numbers, Math. Comput. Modelling, 52, 1763-1770.

[28] Tuglu, N., Kocer, E. G., \& Stakhov, A. P. (2011). Bivariate Fibonacci-like p-polynomials, Appl. Math. Comput., 217 (24), 10239-10246.

[29] Wolfram Research, (2014). Inc Mathematica, Version 10.0: Champaign, Illinois. 